\title{
Correlation between Perceived Fear of COVID-19 Infection and Demand for COVID-19 Vaccination in Thailand: A Cross Sectional Study
}

\author{
Sompoom Sunpaweravong
}

Triam Udom Suksa School, Bangkok, Thailand

\section{ABSTRACT}

As the global COVID-19 pandemic became increasingly severe, the perceived fear of infection amongst the general population increased significantly. Many aspects of COVID-19 infection such as the ailment's severe symptoms and risk of death contributed greatly to the collective fear surrounding this disease. The fear of infection resulted in lifestyle changes for many which in turn caused a great extent of socioeconomic impacts on both the regional and global level. Correlating closely to the fear of infection, vaccine related fear became a growing trend amongst the public. The majority of those fearing the vaccines were skeptical of its safety and risk of severe although rare side effects such as blood clots or convulsion not to mention the more common side effects such as high fevers that deterred a significant portion of the population from getting vaccinated. With these two closely related fears causing great effects on our society, our study decided to study the correlation and interaction between these two metrics. Through measuring both COVID-19 infection and vaccination fear of 351 subjects from diverse demographics, our study found many correlations between the perceived fear for COVID 19 infection, demand and reluctance for COVID 19 vaccination and other factors such as the age of the subject. Our study also took various measurements regarding the subjects involved in this study such as the subject's age and underlying diseases that are known to augment the severity of COVID-19 symptoms such as Diabetes, underlying heart disease and lung diseases. After rigorous data analysis, the study found proportionate correlations between the levels of perceived fear of COVID-19 infection and the demand for vaccination. The demographics of participants (age and underlying diseases) were also found to have a correlative effect on the former measurements.

Keywords: COVID-19 fear, vaccine hesitancy, COVID-19 risk factors

\section{INTRODUCTION}

The outbreak of COVID-19 which was caused by severe acute respiratory syndrome coronavirus 2 (SARS-CoV-2) initially began in December 2019. Soon after its detection in Wuhan, China, the novel coronavirus quickly spread to many other countries worldwide. The rapid spread of the virus eventually turned into a global pandemic which caused socioeconomic effects on many sectors. [1] As the global COVID 19 pandemic became increasingly severe, the perceived fear of infection amongst the general population increased significantly.[2] Many aspects of COVID 19 infection such as the ailment's severe symptoms and risk of death contributed greatly to the collective fear surrounding this disease.

The fear of infection resulted in lifestyle changes for many which in turn caused a great extent of socioeconomic impacts on both the regional and global level. Correlating closely to the fear of infection, vaccine related fear became a growing trend amongst the public. A study found $59.2 \%$ of the population were skeptical of the vaccine's safety and $44.5 \%$ were hesitant in the efficacy of the 
vaccines.[3] With the varying metrics of perceived fear of infection, vaccination demand, age of the subjects and underlying diseases that the subjects had, this research aimed to study the correlation between the factors and highlight significant relations found with each aspect of the measurements. The specificity of the survey was also intended to determine which aspects of COVID-19 infection fear were prevalent and correlated with the demand for COVID-19 vaccination.

\section{MATERIALS AND METHODS}

The cross sectional study collected data from 351 Thai subjects through online surveying. The surveys were distributed through online social media platforms (Line and Facebook) and targeted the Thai population. Information regarding the demographics (age, underlying disease) of each participant were also collected along with questions measuring the perceived fear levels regarding COVID-19 infection and the demand for vaccination. Questions regarding the latter two aspects asked participants to rate their fear or demand of a specific aspect on a scale of 1-5 (with 1 being the lowest and 5 being the highest).

The survey adopted a system for measuring perceived fear of COVID-19 infection by categorizing questions to measure fear on various aspects of COVID19 infection. This study shared some of the fear categories with Karlsson LC et al's study. [4] However, several modifications were made to better suit the current status quo of the COVID-19 pandemic in Thailand.

Table 1: Survey questions measuring the perceived risks and fear of COVID-19 infection

\begin{tabular}{|l|l|}
\hline \multicolumn{1}{|c|}{ Aspect of Measurement } & \multicolumn{1}{c|}{ Question } \\
\hline Perceived likelihood of infection & How much risk do you think you have of being infected with COVID-19? \\
\hline Perceived severity: General & How severe do you think the COVID-19 outbreak in Thailand is? \\
\hline Perceived severity: Personal & How severe do you think your symptoms would be if you contracted COVID-19? \\
\hline Perceived likelihood of death & How likely do you think a COVID-19 patient will die as a result of the disease? \\
\hline Worry: fall ill & How much do you worry about being infected with COVID-19? \\
\hline Worry: death & How much do you worry about dying as a result of COVID-19 infection? \\
\hline Worry: transmission & How much do you worry about potentially spreading COVID-19 to others? \\
\hline
\end{tabular}

Table 2: Survey questions measuring demand and opinions regarding COVID-19 vaccination.

Aspect of Measurement

\begin{tabular}{|l|}
\hline Vaccination demand \\
\hline Tolerance to vaccination risks \\
\hline Vaccination worthiness
\end{tabular}

Vaccination worthiness
Question

How likely are you to take COVID-19 vaccination provided by the government?

To what extent do you think the side effects of COVID-19 vaccination are safe and tolerable?

Do you think the risks associated with vaccination side effects are worth the immunity gained from COVID-19 vaccination?
The study also surveyed underlying diseases which are known to augment the severity of COVID-19 symptoms among the subjects. The list of diseases were based upon guidelines published by the Center for Disease Control and Prevention (CDC). [5] The diseases surveyed among the subjects were Hypertension, Coronary Heart Disease, Diabetes, Chronic Kidney Disease, Asthma, Liver Cirrhosis, Immunodeficiency and Obesity (BMI 30+).

\section{RESULTS}

After collecting data from 351 subjects, the survey received responses from a wide range of demographics.
Table 3: Age range distribution of the studied subjects

\begin{tabular}{|l|c|c|}
\hline \multicolumn{1}{|c|}{ Subject Age Range } & n & \% \\
\hline Below 18 years old & 37 & 10.5 \\
\hline 18-29 years old & 81 & 23.1 \\
\hline 30-39 years old & 82 & 23.4 \\
\hline 40-49 years old & 83 & 23.6 \\
\hline 50-59 years old & 43 & 12.3 \\
\hline Over 60 years old & 25 & 7.1 \\
\hline
\end{tabular}

Table 4: Numbers and proportions of subjects with underlying diseases known to augment COVID-19 severity.

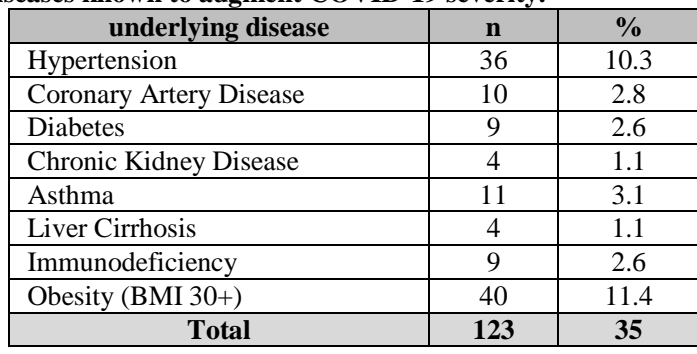


The results from data collection showed a moderate overall trend for fear of COVID-19 infection. There were some significant features among the values, such as the high level of perceived general severity which reflected the severity of the COVID-19 outbreak in Thailand.

Table 5: Results from questions measuring various aspects of perceived fear and worries related to COVID-19 infection (rated on a scale of 1-5)

\begin{tabular}{|l|c|c|}
\hline \multicolumn{1}{|c|}{ Aspect of Measurement } & $\overline{\mathbf{x}}$ & $\boldsymbol{\sigma}$ \\
\hline Perceived likelihood of infection & 3.11 & 1.02 \\
\hline Perceived severity: General & 4.08 & 0.85 \\
\hline Perceived severity: Personal & 3.17 & 0.89 \\
\hline Perceived likelihood of death & 3.30 & 1.00 \\
\hline Worry: fall ill & 3.72 & 0.99 \\
\hline Worry: death & 3.45 & 1.15 \\
\hline Worry: transmission & 3.56 & 1.25 \\
\hline
\end{tabular}

The demand and opinions toward vaccination were also generally moderate. With the Vaccination Demand aspect being rated the highest on average. The collected data also had a relatively wider range of distribution compared to perceived fear levels. With all of the vaccination demand categories possessing a standard deviation $(\sigma)$ of greater than 1

Table 6: Results from questions measuring demand for COVID-19 vaccination (rated on a scale of 1-5).

\begin{tabular}{|l|c|c|}
\hline \multicolumn{1}{|c|}{ Aspect of Measurement } & $\overline{\mathbf{x}}$ & $\boldsymbol{\sigma}$ \\
\hline Vaccination Demand & 3.60 & 1.32 \\
\hline Tolerance to Vaccination Risks & 3.19 & 1.00 \\
\hline Vaccination Worthiness & 3.24 & 1.08 \\
\hline
\end{tabular}

Furthermore, the study measured correlations between different aspects of COVID-19 infection fear and the demand for vaccination using Pearson's Correlation Coefficient as a measurement tool.[6] Another area of study was the correlation between the subject's age, gender and underlying diseases to the perceived fear of COVID-19 infection and the demand for vaccination.

Table 7: Pearson's correlations measured between aspects of COVID-19 infection fear and the demand for vaccination.
\begin{tabular}{|l|c|c|c|}
\hline \multicolumn{1}{|c|}{ Aspects } & Vaccination Demand & Tolerance to Vaccination risks & Vaccination Worthiness \\
\hline Perceived likelihood of infection & 0.24 & 0.16 & 0.20 \\
\hline Perceived severity: General & 0.14 & 0.13 & 0.11 \\
\hline Perceived severity: Personal & 0.09 & 0.07 & 0.11 \\
\hline Perceived likelihood of death & 0.14 & 0.12 & 0.14 \\
\hline Worry: fall ill & 0.22 & 0.12 & 0.16 \\
\hline Worry: death & 0.16 & 0.09 & 0.13 \\
\hline Worry: transmission & 0.11 & 0.05 & 0.07 \\
\hline
\end{tabular}

Note: Pearson's r correlations. Statistically significant correlations $(p<0.05)$ are written in bold letters.

\section{DISCUSSION}

After analyzing correlations between different aspects of COVID-19 infection fear and the demand for vaccination. Multiple key correlations were drawn between these two factors. The strongest correlation being the perceived likelihood of infection with vaccination demand which had a Pearson's correlation coefficient of 0.24 . This was in line with the hypothesis which was based upon the decreased complacency towards vaccination when the prevalence and risks of a certain disease grew. [7]

This same researched principle also explained the second most significant correlation between the worry of falling ill and the demand for COVID-19 vaccination. When a disease is likely to cause severe symptoms, the hesitancy surrounding that disease's vaccine will naturally decrease leading to higher demand for vaccination. Other notable correlations found included vaccination worthiness to worry of falling ill $(\mathrm{r}=0.16)$. There may be many factors affecting vaccine hesitancy such as fear of the side effects or skepticism surrounding the efficacy of these vaccines [8] however, the significant socioeconomic impacts from the COVID-19 pandemic may contribute to the reduction of vaccine hesitant ideologies in individuals. As it was found in this study, subjects who worried more about falling ill or dying as a result of COVID-19 were more likely to demand and be willing to get vaccinated than those who worried and feared infection less.

A notable finding in this study is the lack of a strong correlation and statistical significance between the worry of 
transmitting COVID-19 to the demand for vaccination $(\mathrm{r}=0.11 p>0.05)$. This was the second weakest correlation to demand for vaccination, only stronger than the correlation of the perceived personal severity. This correlation was also statistically insignificant since the $p$ value was more than 0.05 . The weakest correlation in this study, between perceived severity on the individual level to tolerance to vaccination risks also contrasted with the hypotheses and showed a weak and statistically insignificant correlation $(\mathrm{r}=0.07)$

In addition to correlations between perceived fear of COVID-19 infection, this study also discovered a correlation between age and perceived fear of infection. On average, older subjects were found to have a higher level of perceived fear of COVID-19 infection with the 60 years old and older category averaging the highest level of perceived fear. The strongest correlation found in this area was between age and the worry of transmission $(r=0.48 p<0.05)$.

Underlying diseases were also found to have an effect on the perceived fear of infection with many of those diagnosed with underlying diseases known to exacerbate COVID-19 symptoms having a higher level of perceived fear for infection. Vaccination demand was observably higher in subjects that had these underlying diseases with diabetes patients showing the strongest demand for vaccination out of all demographics of subjects.

\section{CONCLUSION}

This study discovered the correlations between different aspects of COVID-19 infection fear and the demand for vaccination. The strongest correlation found was between the perceived likelihood of infection and demand for vaccination $(\mathrm{r}=0.24)$. Statistical significance was also used to determine the weight of each correlation. Furthermore, correlations between subject's age and underlying diseases were also found to correlatively affect the perceived fear of infection and the demand for vaccination. We hope to make positive impacts on society with this study by allowing the fear and opinions toward COVID-19 infection and vaccination to be better understood. With this data attained, we hope to be of use to future studies, strategies and policy planning processes.

Acknowledgements: The author would like to thank Associate Professor Rasmon Kalayasiri, Department of Psychiatry, Faculty of Medicine, Chulalongkorn University for providing the conception for this study. The author would also like to thank Associate Professor Somkiat Sunpaweravong, Department of Surgery, Faculty of Medicine, Prince of Songkla University for editing this manuscript.

\section{Conflicts of interest: None}

\section{Source of funding: None}

\section{Ethical Approval: Approved}

\section{REFERENCES}

1. World Health Organization. Coronavirus disease (COVID-19). Situation Report-130. 2020 May [cited 202131 July]. In: World Health Organization [Internet]. Available from: https://www.who.int/docs/defaultsource/coronaviruse/situationreports/20200529-covid-19-sitrep130.pdf?sfvrsn=bf7e7f0c_4

2. Ornell F, Schuch JB, Sordi AO et al. "Pandemic fear" and COVID-19: mental health burden and strategies. Braz J Psychiatry. 2020;42(3):232-235. doi: 10.1590/1516-4446-2020-0008. Epub 2020 Apr 3. Erratum in: Braz J Psychiatry. 2020;42(3):333. PMID: 32267343; PMCID: PMC7236170.

3. Bono SA, Faria de Moura Villela E, Siau $\mathrm{CS}$ et al. Factors Affecting COVID-19 Vaccine Acceptance: An International Survey among Low- and Middle-Income Countries. Vaccines (Basel). 2021 May 17;9 (5):515. doi: 10.3390/vaccines9050515. PMID: 34067682 ; PMCID: PMC8157062.

4. Karlsson LC, Soveri A, Lewandowsky S et al. Fearing the disease or the vaccine: The case of COVID-19. Pers Individ Dif. 2021 Apr;172:110590. doi: 10.1016/j.paid.2020. 
110590. Epub 2020 Dec 14. PMID: 33518869; PMCID: PMC7832025.

5. COVID-19 Certain Medical Conditions [Internet]:Center for Disease Control and Prevention (US); May 13,2021 [Cited 2021 31 July]. Available from: https://www.cdc.gov/coronavirus/2019ncov/need-extra-precautions/people-withmedical-conditions.html

6. Schober P, Boer C, Schwarte LA. Correlation Coefficients: Appropriate Use and Interpretation. Anesth Analg. 2018 May;126(5):1763-1768. doi: 10.1213/ANE. 0000000000002864 . PMID: 29481436.

7. MacDonald NE; SAGE Working Group on Vaccine Hesitancy. Vaccine hesitancy: Definition, scope and determinants.
Vaccine. 2015 Aug 14;33(34):4161-4. doi: 10.1016/j.vaccine.2015.04.036. Epub 2015 Apr 17. PMID: 25896383.

8. Dubé E, Laberge C, Guay M, Bramadat P, Roy R, Bettinger J. Vaccine hesitancy: an overview. Hum Vaccin Immunother. 2013 Aug;9(8):1763-73. doi: 10.4161/hv.24657. Epub 2013 Apr 12. PMID: 23584253; PMCID: PMC3906279.

How to cite this article: Sunpaweravong S. Correlation between perceived fear of Covid-19 infection and demand for Covid-19 vaccination in Thailand, a cross sectional study. International Journal of Science \& Healthcare Research. 2021; 6(3): 345-349. DOI: https:// doi.org/10.52403/ijshr.20210759 